\title{
Expression of a WIN/SHN-type regulator from wheat triggers disorganized proliferation in the Arabidopsis leaf cuticle
}

\author{
K. JÄGER ${ }^{1}$, A. MISKÓ ${ }^{2}$, A. FÁBIÁN ${ }^{1}$, C. DEÁK ${ }^{2}$, E. KISS-BÁBA ${ }^{2}$, D. POLGÁRI ${ }^{1}$, B. BARNABÁS ${ }^{1}$, \\ and I. PAPP ${ }^{2 *}$ \\ Agricultural Institute, Centre for Agricultural Research, Hungarian Academy of Sciences, \\ H-2462 Martonvásár, Hungary ${ }^{1}$ \\ Department of Plant Physiology and Plant Biochemistry, Faculty of Horticultural Science, Corvinus University of \\ Budapest, $H-1118$ Budapest, Hungary ${ }^{2}$
}

\begin{abstract}
Based on information from the Arabidopsis model system, a putative transcriptional activator of cuticle formation (TaSHN1) was selected among the expressed sequence tags in wheat (Triticum aestivum L.). RT-PCR indicated the preferential expression of this gene in the basal, but not in the middle parts of wheat leaves. This leaf region is a likely site of cuticle formation in cereals. TaSHN1 was cloned and expressed in Arabidopsis, resulting in shiny leaf surfaces and the overproliferation of cuticular material, as observed by electron microscopy. Unlike the Arabidopsis WAX INDUCER/SHINE1 (WIN/SHN1) gene, TaSHN1 triggered disorganized cuticular ultrastructure in the transgenic leaves, with the continuous layers replaced by large electrodense bodies embedded in amorphous lipid material. Toluidine blue staining and dark-adapted water release indicated increased cuticular permeability in TaSHN1-expressing Arabidopsis leaves. The expression of TaSHN1 resulted in a moderate decrease of the total number of stomata per unit leaf area in comparison with the wild type. Drought tolerance of Arabidopsis was unaffected by the transgene. The data indicate that this putative wheat orthologue of WIN/SHN transcription factors (TaSHN1) elicited both overlapping and new, distinctive phenotypes compared to other WIN/SHN-overexpressing plants. TaSHN1 transgenic Arabidopsis lines should provide a rich source of material for further comparative biochemical, physiological, and genetic studies.
\end{abstract}

Additional key words: cutin, drought tolerance, stomata, Triticum aestivum, wax, WIN/SHN transcription factors.

\section{Introduction}

Besides stomatal transpiration (Sirichandra et al. 2009), the cuticle contributes significantly to water loss especially under drought (Riederer and Schreiber 2001). According to the work of Rawson and Clarke (1988), the cuticular component represents a substantial portion of total water loss from wheat leaves under water-stressed conditions. Genetic determinants for the biosynthesis of cuticular material have been described most extensively in model species (Kunst and Samuels 2009). Among these genes a growing number of transcriptional regulators have been identified (Borisjuk et al. 2014). The formation of the cuticle in Arabidopsis is affected by the WAX INDUCER/SHINE (WIN/SHN) clade of
APETALA2/ethylene-responsive elements binding protein (AP2/EREBP) transcription factors (Aharoni et al. 2004, Broun et al. 2004) with an implicated function in cutin biosynthesis (Kannangara et al. 2007).

A few genes responsible for cuticular lipid biosynthesis have been uncovered in cereals (e.g., Yu et al. 2008, Hu et al. 2010a, Wu et al. 2011), whereas no transcriptional regulator of these processes has been functionally identified in wheat.

The main goal of the present study was the identification and functional characterization of a potential regulator of cuticle formation in wheat leaves. A putative regulator gene was selected based on sequence

Submitted 3 December 2013, last revision 22 May 2014, accepted 20 August 2014.

Abbreviations: PPDF - photosynthetic photon flux density; RT-PCR - reverse transcription - polymerase chain reaction; RWC - relative water content; WIN/SHN - WAX INDUCER/SHINE.

Acknowledgements: The authors wish to thank E. Kapral Bék and E. Gondos for their excellent technical assistance. The work was supported by the TÁMOP 4.2.1/B-09/1/KMR/-2010-0005, NKTH-OTKA CK80211, and NKTH-OTKA CK80274 grants from the National Development Agency of Hungary and the 00044/11 Bolyai János Research Scholarship of the Hungarian Academy of Sciences. C. Deák was financially supported by the Doctoral Council of Life Sciences of Corvinus University of Budapest.

* Corresponding author; fax: (+36) 1209 6388; e-mail: istvan.papp2@uni-corvinus.hu 
homology to a member of the WIN/SHN clade of transcription factors, as structure and function of this gene family have been already shown conserved in several species (e.g., Zhang et al. 2005). Our hypothesis was that the selected wheat gene might be functionally related to other members of the gene family described in

\section{Materials and methods}

Plants and growth conditions: For expression profiling and cloning TaSHN sequences, four winter wheat (Triticum aestivum L.) cultivars (Plainsman V, Mv Emese, GK Élet, and Cappelle Desprez) were germinated in a soil, sand, and peat $(60+20+20 \%)$ mixture and grown in $P G R-15$ phytotron chambers (Conviron, Winnipeg, Canada) at a max/min temperatures of $21 / 14{ }^{\circ} \mathrm{C}$, a relative humidity of $75 / 65 \%$, a 12-h photoperiod, and a photosynthetic photon flux density (PPFD) of $500 \mu \mathrm{mol} \mathrm{m} \mathrm{m}^{-2} \mathrm{~s}^{-1}$ until the 3-leaf stage (21 d after emergence) (Tischner et al. 1997). Cloning TaSHN sequences was carried out using cDNA derived from cv. Cappelle Desprez. Irrigation was carried out regularly in the morning. The covered parts of the $3^{\text {rd }}$ leaves, from the base to the point of leaf emergence, were separated and used for RNA extraction. Samples were also taken from the middle of emerged $3^{\text {rd }}$ leaves. To clone cDNA of the TaSHN1 gene, seeds of Mv Emese were germinated in Petri dishes lined with moistened filter paper at $21{ }^{\circ} \mathrm{C}$, a $12-\mathrm{h}$ photoperiod, and PPDF of $150 \mu \mathrm{mol} \mathrm{m} \mathrm{m}^{-2} \mathrm{~s}^{-1}$. After germination, the seedlings were grown under the same conditions for $10 \mathrm{~d}$ before RNA extraction. Arabidopsis thaliana L. cv Columbia plants were cultivated in soil (CompoSana type II, Compo, Münster, Germany) in growth chambers (Conviron) at $21{ }^{\circ} \mathrm{C}$, a relative humidity of $60 \%$, and PPFD of $120 \mu \mathrm{mol} \mathrm{m} \mathrm{m}^{-2} \mathrm{~s}^{-1}$. Short-day (a 10-h photoperiod) was applied for four weeks after sowing and then it was changed to long-day (a 14-h photoperiod).

Toluidine Blue staining and water release in dark: Toluidine Blue, a hydrophilic dye, can penetrate through discontinuous or defective plant cuticles, which has made this stain a useful tool for characterization of cuticular mutants (Tanaka et al. 2004, Weng et al. 2010). Leaves of six-week-old Arabidopsis plants were treated with Toluidine Blue as described by Tanaka et al. (2004) with some modifications. Whole rosette leaves were immersed in $2.5 \times 10^{-2} \mathrm{~g} \mathrm{dm}^{-3}$ Toluidine Blue (Sigma, St. Louis, MO, USA) in water and gently agitated at room temperature for $2 \mathrm{~h}$, followed by washing in distilled water to remove excess dye. The water release from six-week-old Arabidopsis plants was measured after dark adaptation according to Kosma et al. (2009). Whole rosettes were saturated with water by gentle agitation in distilled water at room temperature for $1 \mathrm{~h}$. Excess water was removed by blotting, then the water saturated mass (SM) of the samples was measured. Changes in fresh mass (FM) were monitored for a further $160 \mathrm{~min}$ in the other species. In order to obtain indication about the biological processes this gene is potentially involved in, we set off to establish its expression pattern in growing leaves. The effect of this wheat gene on cuticular architecture and function was also studied by monitoring its transgenic expression in the model plant Arabidopsis.

dark. The dry mass (DM) of the rosettes was determined by weighing the samples after drying them overnight at $80{ }^{\circ} \mathrm{C}$. The relative water content (RWC) was calculated using the following equation:

$\mathrm{RWC}[\%]=[(\mathrm{FM}-\mathrm{DM}) /(\mathrm{SM}-\mathrm{DM})] \times 100$

Drought tolerance of Arabidopsis plants: TaSHN1transformed and control Arabidopsis plants were grown in pots in phytotron chambers as described above. On the first day of the experiments, the soil was saturated with water to field capacity. In order to induce water stress, no further irrigation was applied until the end of the drying period. Soil water content was monitored by the gravimetric method. The pots were weighed in the early afternoon of the days indicated during the measurement period. Irrigation was resumed by bringing the pots to field capacity with excess water. At the end of the experiment, the content of the pots were kept at $80{ }^{\circ} \mathrm{C}$ for $24 \mathrm{~h}$ to achieve total desiccation of the soil. The gravimetric water content (GWC) was calculated as follows: $\mathrm{GWC}[\%]=[(\mathrm{Ms}-\mathrm{DMs}) /(\mathrm{FMs}-\mathrm{DMs})] \times 100$, where; Ms - the mass of pot at the time point indicated, DMs - the mass of pot after desiccation, FMs - the mass of pot at field capacity.

Analysis of stomatal density: Fully expanded rosette leaves at developmental stage 6.3 (i.e., $30 \%$ of flowers open; Boyes et al. 2001) were cleared overnight in a solution containing $410 \mathrm{~cm}^{3} \mathrm{dm}^{-3}$ ethanol, $210 \mathrm{~cm}^{3} \mathrm{dm}^{-3}$ chloroform, $170 \mathrm{~cm}^{3} \mathrm{dm}^{-3}$ lactic acid, $210 \mathrm{~cm}^{3} \mathrm{dm}^{-3}$ phenol (dissolved in distilled water 50:50), and $3.63 \mathrm{M}$ chloral hydrate, washed, and stored in $50 \%(\mathrm{v} / \mathrm{v})$ ethanol. The cleared epidermal peels were manually dissected from the middle parts of the specimens using forceps, flattened in distilled water, mounted on microscope slides in $500 \mathrm{~cm}^{3} \mathrm{dm}^{-3}$ glycerol in water and examined under an Olympus B51 microscope (Olympus, Tokyo Japan). Four photographs of each of the peels dissected from both adaxial and abaxial sides of the rosette leaves were taken. Three leaves of three plants were analyzed for each genotype, and the experiment was repeated twice. Stomatal density was determined using an ImagePro Plus 4.5 image analysis system (Media Cybernetics, Silver Spring, MD, USA).

Transmission electron microscopy: For transmission electron microscopy, three rosette leaves (cut into $1 \mathrm{~mm}^{2}$ segments) from three Arabidopsis thaliana plants of each genotype were fixed for $4 \mathrm{~h}$ at room temperature in a 
primary fixative containing $25 \mathrm{~cm}^{3} \mathrm{dm}^{-3}$ glutaraldehyde in a $0.05 \mathrm{M}$ Na-cacodylate buffer, $\mathrm{pH} 7.2$ (CB). After fixation, the samples were washed in $\mathrm{CB}$ and post-fixed in $10 \mathrm{~g} \mathrm{dm}^{-3}$ osmium tetroxide in $0.05 \mathrm{M} \mathrm{CB}$ at $4{ }^{\circ} \mathrm{C}$ for $3 \mathrm{~h}$. After washing in $\mathrm{CB}$, the samples were dehydrated through a gradient series of ethanol concentrations (10, 30, 50, 70, 90, and $100 \%$ ), infiltrated with Spurr's embedding medium (Spurr 1969) according to the manufacturer's instructions (Sigma-Aldrich, St. Louis, MO, USA), and polymerized at $60^{\circ} \mathrm{C}$ for $48 \mathrm{~h}$. Ultrathin sections were cut using an Ultracut $E$ microtome and mounted on Formvar-coated (SPI-Chem, West Chester, PA, USA) 100-mesh nickel grids stained with $30 \mathrm{~g} \mathrm{dm}^{-3}$ aqueous uranyl acetate and $0.8 \mathrm{~g} \mathrm{dm}^{-3}$ lead citrate. The sections were examined under a Zeiss EM-910 (Wetzlar, Germany) electron microscope at $80 \mathrm{kV}$.

Scanning electron microscopy: For scanning electron microscopy, parts of three rosette leaves from three Arabidopsis thaliana plants of each genotype were fixed in $28 \mathrm{~g} \mathrm{dm}^{-3}$ glutaraldehyde in a 0.1 M HEPES buffer $(\mathrm{pH}$ 7.2) at $4{ }^{\circ} \mathrm{C}$ for $3 \mathrm{~h}$, washed in the HEPES buffer and post-fixed in aqueous $10 \mathrm{~g} \mathrm{dm}^{-3}$ osmium tetroxide for $3 \mathrm{~h}$. After rinsing in the HEPES buffer, the tissues were dehydrated in the same graded ethanol series, criticalpoint dried (CPD 030, BAL-TEC, Balzers, Liechtenstein), mounted on nickel grids, and coated with gold (SCD 005, $B A L-T E C)$. The leaf surfaces were examined under a Zeiss EM-910 electron microscope at $80 \mathrm{kV}$.

RNA preparation, cDNA synthesis, and RT-PCR: RNA was isolated from leaves with Tri Reagent (Molecular Research Center, Cincinnati, OH, USA) according to the instructions of the manufacturer. A first strand cDNA synthesis kit (Fermentas, Vilnius, Lithuania) was used for the reverse transcription of the RNA samples. RT-PCR was performed with a Advantage-GC2 polymerase mix (Clontech, Mountain View, CA, USA), applying a total of 30 cycles. The genespecific primers used are listed in Table 1. An equal use of cDNA templates was confirmed by RT-PCR with the control primers TalKa and TalKb amplifying sequence Ta2776 (Table 1), as suggested by Paolacci et al. (2009). RT-PCR was repeated three times on different biological samples with at least two technical repetitions in each case.

Recombinant DNA: Basic recombinant techniques were performed as described by Sambrook et al. (1989) using the TOP10 Escherichia coli strain (Invitrogen, Carlsbad, CA, USA). The enzymes were purchased from

\section{Results}

The primer pairs were designed for RT-PCR amplification and cloning the predicted full length of the TaSHN1 coding sequence (buSWk1/2, Table 1). The plant material was collected from the basal parts of the $3^{\text {rd }}$
Fermentas. The RT-PCR products were purified using an Illustra GFX PCR DNA and gel band purification kit ( $G E$ Healthcare, Uppsala, Sweden). The pBluescriptII KS and $p B C S K$ vectors were obtained from Stratagene (La Jolla, CA, USA). The $p$ GreenII0179 binary vector (Hellens et al. 2000) was modified by providing it with the $35 \mathrm{~S}$ promoter and $35 \mathrm{~S}$ terminator sequences of the Cauliflower Mosaic Virus. The Agrobacterium tumefaciens strain GV3101 (Koncz and Schell 1986) was used for plant transformation.

Plant transformation: Arabidopsis thaliana cV Columbia plants were transformed using the vacuum infiltration method (Zhang et al. 2006). For selection in transformation experiments, Arabidopsis plants were grown in vitro on half-strength MS media (Murashige and Skoog 1962) supplemented with $5 \times 10^{-2} \mathrm{~g} \mathrm{dm}^{-3}$ Hygromycin under a $12-\mathrm{h}$ photoperiod and PPFD of $150 \mu \mathrm{mol} \mathrm{m} \mathrm{m}^{-2} \mathrm{~s}^{-1}$. Hygromycin resistant seedlings were grown further under phytotron conditions as described above.

Identification of TaSHN1: The Arabidopsis thaliana WIN/SHN1 sequence (NP_172988.1) was used as the query in a $t B L A S T n$ search (Altschul et al. 1990) of the Triticum aestivum subset of the NCBI expressed sequence tag (EST) database. The best hits obtained in the search were members of Ta31753 and Ta44806 wheat Unigenes. A further search of the Triticeae Full-Length CDS DataBase (Mochida et al. 2009) with sequences from these Unigenes identified accession tplb0011g14 which codes for a putative protein 227 amino acids long. This gene, referred to hereafter as TaSHN1, exhibited the greatest similarity to wheat Unigene Ta44806. The encoded protein was found to be 58.1 and $72.8 \%$ identical with Arabidopsis WIN/SHN1 (Aharoni et al. 2004, Broun et al. 2004) and the orthologous rice OsWR1 transcription factor (Wang et al. 2012), respectively (Fig. 1 Suppl.).

The primers were designed with the Primer Premier software (Premier Biosoft, Palo Alto, CA, USA). Multiple sequence alignment was performed using the CLUSTAL $W$ (v. 2.1) program (Chenna et al. 2003). Primary DNA sequences were determined by Biomi Kft (Gödöllö, Hungary).

Statistical analysis: All data are pooled means from the replicates and were statistically evaluated using the Student's $t$-test and balanced analysis of variance ANOVA (SPSS for Windows, version 10.0).

leaf covered by the sheath, and from the emerged regions of the same blades, pooled from 2 - 3 individual plants for each RNA extraction. cDNAs were prepared and found to be devoid of genomic DNA contamination, as 


\section{K. JÄGER et al.}

Table 1. Primer sequences used for the amplifications of TaSHN1 (buSWk1 and buSWk2) and Ta.2776 (control primers TalKa and TalKb; Paolacci et al. 2009) in RT-PCR reactions.

\begin{tabular}{ll}
\hline Primer name & Primer sequence \\
\hline buSWk1 & 5'-AGAGGATCCAACAATGGTACAGTCCAAGAAGAAGTTTCGC-3' \\
buSWk2 & 5'-GTGGAATTCTCAGATGACGAGGCTGCCTTCTTCACCG-3' \\
TalKa & 5'-GTAGCATTATGTTTGTGCCTTG-3' \\
TalKb & 5'-GGAGAGCCAGTCAAGACCCTCG-3'
\end{tabular}

revealed by intron spanning control RT-PCR reactions (data not shown). The TaSHN1 transcript was found to be expressed in the basal but not in the emerged leaf regions (Fig. 1). Biological and technical repetitions gave very similar results.

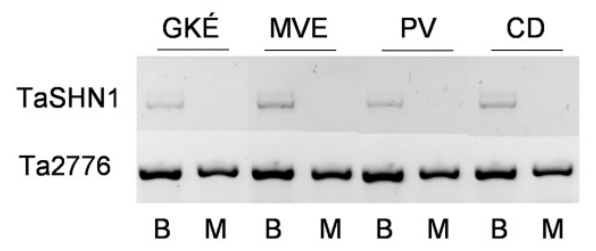

Fig. 1 The TaSHN1 transcription in the basal (B) and middle (M) leaf regions in different wheat cultivars (GKÉ - GK Élet, MVE - MV Emese, PV - Plainsman V, and CD - Cappelle Desprez). For further details, see the text. Ta2776 - a reference gene.

Amplified DNA fragments from the RT-PCR reaction of Cappelle Desprez samples (from the leaf base) were cloned into the $p$ BluescriptII $K S$ vector using BamHI and EcoRI restriction enzymes. A high sequence homology to tplb0011g14 from the Triticeae Full-Length CDS DataBase and to ESTs of Unigene Ta44806 was revealed by sequencing eight independent clones.

The cDNA of TaSHN1 was cloned from cv. MvEmese into $p B C S K$ and further into a modified pGreenII0179 vector (Hellens et al. 2000) with BamHI and EcoRI restriction enzymes. This construct was introduced into Agrobacterium and transformed into Arabidopsis. Transgenic plants were selected on a half- strength MS medium supplemented with hygromycin and were grown till maturity in soil. Eight independent transgenic lines were recovered, most of which ( 7 lines) exhibited strikingly shiny leaf surfaces (Fig. 2A,B). The presence and expression of TaSHN1 in the transgenic Arabidopsis lines were confirmed by PCR and RT-PCR.

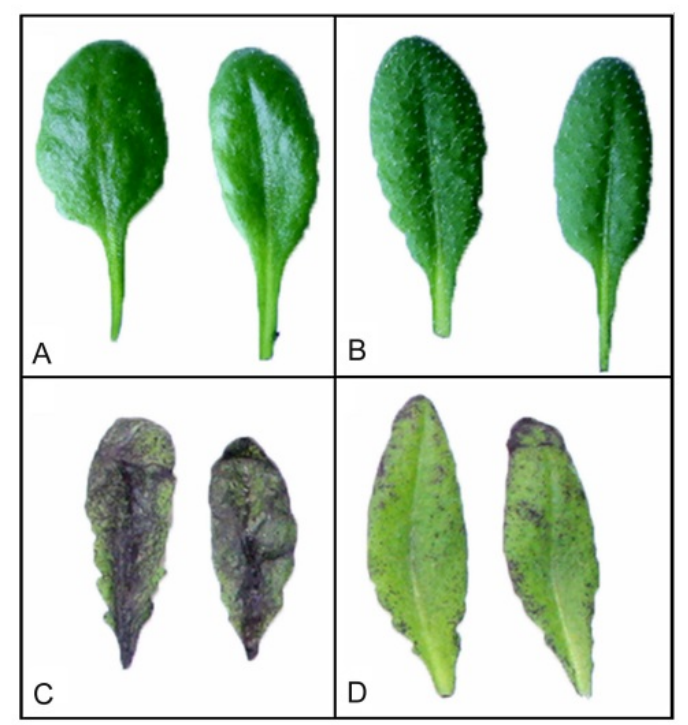

Fig. 2. A - Rosette leaves of four-week-old TaSHN1 transgenic Arabidopsis plants (line \#4/2) exhibiting shiny surfaces. B - A wild-type control (Columbia ecotype). C - Toluidine Blue stained leaves of six-week-old TaSHN1 transgenic Arabidopsis plants (line \#4/2). D - Toluidine Blue hardly stained leaves of the wild-type.
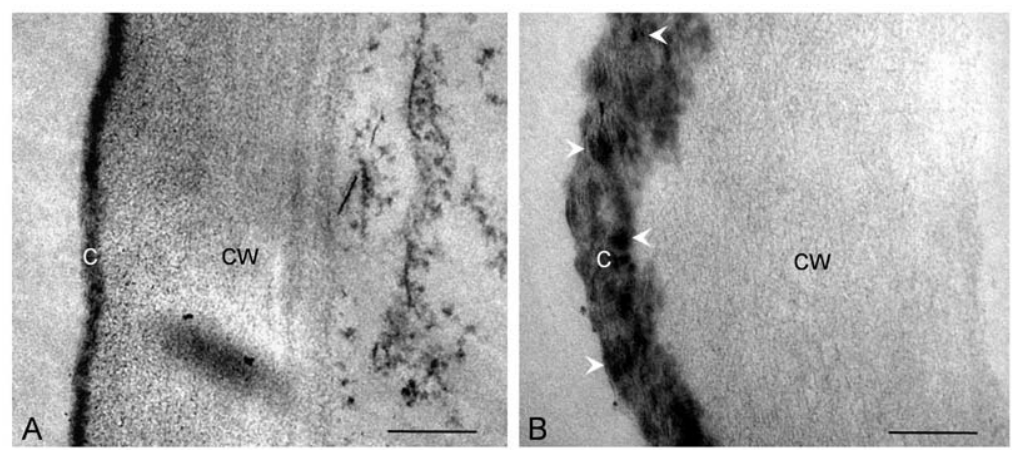

Fig. 3 Ultramicrographs of wild type $(A)$ and Col-TaSHN1-4/2 (B) A. thaliana pavement cell cuticles. Note a high number of amorphous electrodense intracuticular inclusions (the arrows) in Col-TaSHN1-4/2 genotype (c - cuticle layer, cw - cell wall; the $b a r=200 \mathrm{~nm}$ ). 
The co-segregation of the shiny phenotype and the transgene was confirmed, a line homozygous for the transgene was developed (Col-TaSHN1-4/2)and used in further experiments.

Scanning electron microscope observations revealed that the morphology of epicuticular wax changed significantly in Col-TaSHN1-4/2 line compared to the wild-type control. In contrast to the smooth surface of the wild type (Fig. 2A,C Suppl.), platelet-like epicuticular wax depositions were discernible on both the abaxial (Fig. 2B Suppl.) and adaxial surfaces (Fig. 2D Suppl.). The image analysis of the transmission electron micrographs confirmed that the cuticle of Col-TaSHN1$4 / 2$ plants was significantly thicker than that of the wildtype plants (Table 2). Moreover, a large number of amorphous electrodense intracuticular inclusions were found in the cuticle of Col-TaSHN1-4/2 plants, which were not observed in the wild type (Fig. 3).

Table 2. Cuticle thickness [nm] of abaxial and adaxial leaf epidermises. Means \pm SE, $n=$ ??. Different letters indicate significant differences at $P \leq 0.05$.

\begin{tabular}{lrr}
\hline Genotype & Abaxial & \multicolumn{1}{l}{ Adaxial } \\
\hline Col-TaSHN1-4/2 & $158.3 \pm 75.7^{\mathrm{a}}$ & $144.0 \pm 47.5^{\mathrm{a}}$ \\
Wild type & $39.3 \pm 10.2^{\mathrm{b}}$ & $39.3 \pm 13.3^{\mathrm{b}}$ \\
\hline
\end{tabular}

The total number of stomata per unit area of leaves (abaxial + adaxial) decreased from $278.3 \pm 46.81 \mathrm{~cm}^{-2}$ (wild type) to $256.4 \pm 46.9 \mathrm{~cm}^{-2}$ (Col-TaSHN1-4/2), which was significantly different at $P \leq 0.05$.

The toluidine blue treatment revealed consistently stronger staining the TaSHN1 transgenic leaves compared to the wild type (Fig. 2C,D). Rosettes of the dark adapted transgenic plants lost water faster than those of the wild type (Fig. 4).

\section{Discussion}

In recent years, genetic determinants of cuticle formation in crop plants have been intensively explored. Some of the genes involved in the biosynthetic processes of cuticular components have been identified and cloned from wheat, rice, and barley (e.g., Yu et al. 2008, Chen et al. 2011). Richardson et al. $(2005,2007)$ found that the expressions of a few candidate cuticle-associated genes parallel the deposition of cuticular material in the basal region of barley leaves.

Among the cereals, WIN/SHN type regulators of cuticle development have been identified in barley (Taketa et al. 2008) and rice (Wang et al. 2012). Kosma et al. (2010) found cuticle-related genes in wheat when studying biotic interactions with the Hessian fly. This study indicates a relationship between the transcript abundance of an MYB30 homologous putative
The WT and TaSHN1-expressing plants were water stressed by withholding irrigation. Signs of wilting first became visible for all the plants after $4-5 \mathrm{~d}$ of water deprivation; the two groups could not be distinguished based on the visible symptoms of water shortage. The GWC values of the two genotypes decreased in parallel with no significant difference throughout the experiment (Fig. 3 Suppl.). By the $10^{\text {th }}$ day of the treatment, all the plants wilted completely without any notable difference in drought hardiness between them (Fig. 4A,B Suppl.). The plants were left without water supplies for an additional week which was followed by re-irrigation. A similar, small fraction of the plants of the two genotypes (4/24 of WT, $3 / 27$ of Col-TaSHN1 4/2) were able to recover after the drought period (Fig. 4C,D Suppl.). The experiment was repeated twice with similar results. A phenotypically more severe transgenic line, also displaying leaf curling and dwarfing (Col-TaSHN1 1/2), was also tested and exhibited similar behavior in comparison with the wild type (data not shown).

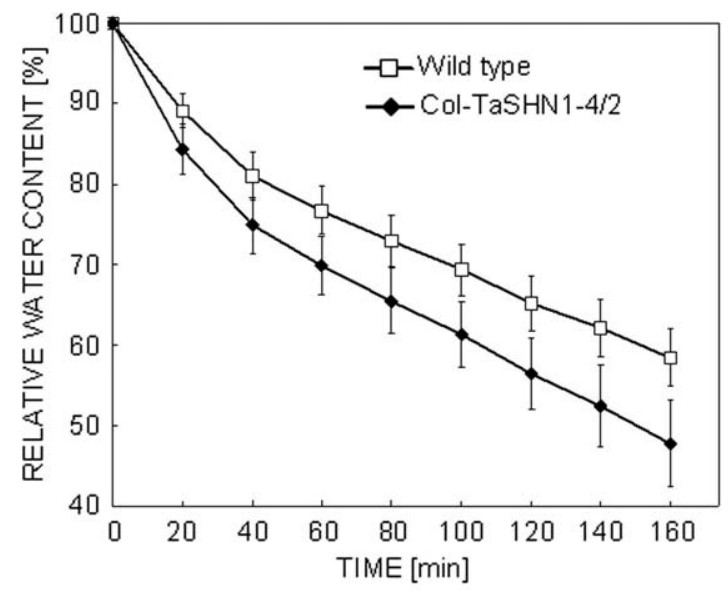

Fig. 4. Water loss from dark-adapted rosettes of wild-type (Columbia) and Col-TaSHN1-4/2 A. thaliana plants.

transcription factor and the production of specific wax constituents. To the best of our knowledge, this is the only transcription factor of wheat that has been associated with the biosynthesis of cuticular components.

In a search for WIN/SHN1 orthologues in wheat sequences, Unigenes Ta31753 and Ta44806 were identified in the NCBI database. The expression profile of Ta44806 is very similar to that of the WIN/SHN genes, leading us to speculate that Unigene Ta44806 might be a WIN/SHN orthologue based on conserved sequences and expression patterns. Accession tplb0011g14 in the Triticeae Full-Length CDS DataBase (Mochida et al. 2009) was found to bear a great resemblance to the above wheat sequences (especially to Unigene Ta44806) and was named TaSHN1. When this gene was analyzed further, its product displayed a greater similarity to 
published rice WIN/SHN1 orthologue OsWR1 (Wang et al. 2012) than to the Arabidopsis WIN/SHN1 protein (Fig. 1 Suppl.). The expression pattern of the gene was investigated in the leaves of four winter wheat genotypes: the drought-tolerant cvs. Plainsman V and Mv Emese, and the drought-sensitive cvs. GK Élet and Cappelle Desprez (Guóth et al. 2009). The transcript accumulation was tested in the middle leaf region and also at the base of the $3^{\text {rd }}$ leaf, still covered by the sheath, where cuticular material is most likely to accumulate. TaSHN1-specific RT-PCR selectively amplified a cDNA fragment from the basal leaf region but not from the middle of the leaves (Fig. 1). This finding implies that TaSHN1 expression might be associated with cuticle formation in the basal leaf region, so the gene may have a great potential impact on the water-sealing ability of this layer in later stages of development. The RT-PCR products from this experiment were cloned and their primary sequences determined, confirming identity.

To reveal the biological function of TaSHN1 in cuticle deposition, its full-length coding sequence, represented by an allele of the Ta44806 wheat Unigene (TriFLDB accession tplb0011g14 - TaSHN1), was cloned and expressed in Arabidopsis. A striking visible phenotype was evident in most transgenic Arabidopsis lines, very similar to that described earlier for $W I N / S H N$ overexpressing plants (Aharoni et al. 2004, Broun et al. 2004). The leaves exhibited a brilliant, shiny appearance, which coincided with a characteristic change in the wax deposition on the leaf surfaces observed by SEM, testifying to the overproduction of cuticular material by the epidermis. In order to further investigate the effects of TaSHN1 expression on the cuticle of Arabidopsis, transmission electron microscopy (TEM) was applied on cross-sections of leaf samples from the transgenic plants. The overproliferation of the cuticle was evident from the TEM results, as the cuticular thickness increased substantially (Fig. 3). The well-organized structure of the cutin matrix of wild-type plants, however, was replaced by a large number of amorphous electrodense intracuticular inclusions appearing in a disorganized manner. These findings partially corresponded to the results of Broun et al. (2004) as far as the increased accumulation of cuticular material is concerned. The wheat gene, however, triggered cuticular development with more aberrant fine architecture than its Arabidopsis counterpart.

Increased cuticular permeability of the transgenic leaf cuticle was found for the cationic dye Toluidine blue
(Fig. 2C,D). A faster water loss from dark adapted leaves was detected in the TaSHN1 transgenic lines compared to the wild type (Fig 4), which was interpreted as indication for increased water permeability of the cuticle. Incomplete closure of stomata is an inherent uncertainty of this type of experiments with the potential danger of overestimating the cuticular component of water loss (Šantrůček et al. 2004). Despite this shortcoming, the method has been successfully used to characterize cuticular permeability for water in model species and also in crop plants. An increased cuticular permeability of the TaSHN1 transgenic plants is coherent with the microstructural changes observed in this layer. Arabidopsis lines overexpressing endogenous WIN/SHN genes (Aharoni et al. 2004, Broun et al. 2004) displayed an increased drought tolerance at the whole plant level. TaSHN1, however, did not confer any drought tolerance or improved recovery to Arabidopsis (Fig. 4 Suppl.). This reinforces that other than cuticular traits should be associated with the induced drought hardiness of several $W I N / S H N$ overexpressors. In fact, recent results testify that WIN/SHN genes may regulate several independent components of abiotic defense responses (Borisjuk et al. 2014), which is a common feature in plant stress signaling ( $\mathrm{Hu}$ et al 2010b). Cuticular traits therefore represent just one facet of the alterations determining the phenotype of WIN/SHN overexpressing plants.

Yang et al. (2011) proposed that a reduced stomatal density may be a decisive factor in improved drought tolerance of AtSHN1-overexpressing Arabidopsis lines. The expression of TaSHN1 triggered an overall decline of $7.9 \%$ in the number of stomata per unit leaf area. This is a modest change compared to the large decrease in stomatal frequency observed by Yang et al. (2011), so our data do not contradict the proposed role of stomatal density in the drought tolerance induced.

The most feasible interpretation of the data presented here is that TaSHN1 plays a role in the formation of the cuticle on wheat leaf surfaces. Besides the identification of a new regulator gene in wheat, our results open new possibilities to analyze mechanisms of cuticular biosynthetic processes operating in Arabidopsis. TaSHN1 elicited unique changes in the ultrastructure as well as in specifically affected permeability of the cuticle in the transgenic plants. This will facilitate comparative studies in order to reveal the biochemical, physiological, and genetic bases of the overlapping but not identical phenotypes of Arabidopsis plants expressing WIN/SHN transgenes of different origin.

\section{References}

Aharoni, A., Dixit, S., Jetter, R., Thoenes, E., Van Arkel, G., Pereira, A.: The SHINE clade of AP2 domain transcription factors activates wax biosynthesis, alters cuticle properties, and confers drought tolerance when overexpressed in Arabidopsis. - Plant Cell 16: 2463-2480, 2004.

Altschul, S., Gish, W., Miller, W., Myers, E., Lipman, D.: Basic

local alignment search tool. - J. mol. Biol. 215: 403-410, 1990.

Borisjuk, N., Hrmova, M., Lopato S.: Transcriptional regulation of cuticle biosynthesis. - Biotechnol. Adv. 32: 526-540, 2014.

Boyes, D.C., Zayed, A.M., Ascenzi, R., McCaskill, A.J., 
Hoffman, N.E., Davis, K.R., Görlach, J.: Growth-stagebased phenotypic analysis of Arabidopsis: a model for high throughput functional genomics in plants. - Plant Cell 13: 1499-1410, 2001

Broun, P., Poindexter, P., Osborne, E., Jiang, C.Z., Riechmann, J.L.: WIN1, a transcriptional activator of epidermal wax accumulation in Arabidopsis. - Proc. nat. Acad. Sci. USA 101: 4706-4711, 2004.

Chen, G., Komatsuda, T., Ma, J.F., Nawrath, C., Pourkheirandish, M., Tagiri, A., Hu, Y.G., Sameri, M., Li, X., Zhao, X., Liu, Y., Li, C., Ma, X., Wang, A., Nair, S., Wang, N., Miyao, A., Sakuma, S., Yamaji, N., Zheng, X., Nevo, E.: An ATP-binding cassette subfamily G full transporter is essential for the retention of leaf water in both wild barley and rice. - Proc. nat. Acad. Sci. USA 108: 12354-12359, 2011.

Chenna, R., Sugawara, H., Koike, T., Lopez, R., Gibson, T.J., Higgins, D.G., Thompson, J.D.: Multiple sequence alignment with the Clustal series of programs. - Nucl. Acids Res. 31: 3497-3500, 2003.

Guóth, A., Tari, I., Gallé, Á., Csiszár, J., Pécsváradi, A., Cseuz, L., Erdei, L.: Comparison of the drought stress responses of tolerant and sensitive wheat cultivars during grain filling: changes in flag leaf photosynthetic activity, ABA levels, and grain yield. - J. Plant Growth Regul. 28: 167-176, 2009.

Hellens, R.P., Edwards, E.A., Leyland, N.R., Bean, S., Mullineaux, P.M.: pGreen: a versatile and flexible binary $\mathrm{Ti}$ vector for Agrobacterium-mediated plant transformation. Plant mol. Biol. 42: 819-832, 2000.

Hu, X.J., Zhang, Z.B., Fu, Z.Y., Xu, P., Hu, S.B., Li, W.Q.: Significance of a $\beta$-ketoacyl-CoA synthase gene expression for wheat tolerance to adverse environments. - Biol. Plant. 54: 575-578, 2010a.

Hu, X.J., Zhang, Z.B., Xu, P., Fu, Z.Y., Hu, S.B., Song, W.Y.: Multifunctional genes: the cross-talk among the regulation networks of abiotic stress responses. - Biol. Plant. 54: 213223, 2010b

Kannangara, R., Branigan, C., Liu, Y., Penfield, T., Rao, V., Mouille, G., Höfte, H., Pauly, M., Riechmann, J.L., Broun, P.: The transcription factor WIN1/SHN1 regulates cutin biosynthesis in Arabidopsis thaliana. - Plant Cell 19: 12781294, 2007.

Koncz, C., Schell, J.: The promoter of TL-DNA gene 5 controls the tissue-specific expression of chimeric genes carried by a novel type of Agrobacterium binary vector. - Mol. gen. Genet. 204: 383-396, 1986.

Kosma, D.K., Bourdenx, B., Bernard, A., Parsons, E.P., Lü, S., Joubès, J., Jenks, M.A.: The impact of water deficiency on leaf cuticle lipids of Arabidopsis. - Plant Physiol. 151: 1918-1929, 2009.

Kosma, D.K., Nemacheck, J.A., Jenks, M.A., Williams, C.E.: Changes in properties of wheat leaf cuticle during interactions with Hessian fly. - Plant J. 63: 31-43, 2010.

Kunst, L., Samuels, S.: Plant cuticles shine: advances in wax biosynthesis and export. - Curr. Opin. Plant. Biol. 12: 721727, 2009.

Mochida, K., Yoshida, T., Sakurai, T., Ogihara, Y., Shinozaki, K.: TriFLDB: a database of clustered full-length coding sequences from Triticeae with applications to comparative grass genomics. - Plant Physiol. 150: 1135-1146, 2009.

Murashige, T., Skoog, F.: A revised medium for rapid growth and bioassays with tobacco tissue cultures. - Physiol. Plant. 15: 473-497, 1962.

Paolacci, A.R., Tanzarella, O.A., Porceddu, E., Ciaffi, M.: Identification and validation of reference genes for quantitative RT-PCR normalization in wheat. - BMC mol. Biol. 10: 11, 2009.

Rawson, H.M., Clarke, J.M.: Nocturnal transpiration in wheat. Aust. J. Plant Physiol. 15: 397-406, 1988.

Richardson, A., Franke, R., Kerstiens, G., Jarvis, M., Schreiber, L., Fricke, W.: Cuticular wax deposition in growing barley (Hordeum vulgare) leaves commences in relation to the point of emergence of epidermal cells from the sheaths of older leaves. - Planta 222: 472-483, 2005.

Richardson, A., Wojciechowski, T., Franke, R., Schreiber, L., Kerstiens, G., Jarvis, M., Fricke, W.: Cuticular permeance in relation to wax and cutin development along the growing barley (Hordeum vulgare) leaf. - Planta 225: 1471-1481, 2007.

Riederer, M., Schreiber, L.: Protecting against water loss: analysis of the barrier properties of plant cuticles. - J. exp. Bot. 52: 2023-2032, 2001.

Sambrook, J., Fritsch, E.F., Maniatis, T.: Molecular Cloning: A Laboratory Manual. - Cold Spring Harbor Laboratory Press, Cold Spring Harbor 1989.

Šantrůček J., Šimánová E., Karbulková J., Šimková M., Schreiber L.: A new technique for measurement of water permeability of stomatous cuticular membranes isolated from Hedera helix leaves. - J. exp. Bot. 55: 1411-1422, 2004.

Sirichandra, C., Wasilewska, A., Vlad, F., Valon, C., Leung, L.: The guard cell as a single-cell model towards understanding drought tolerance and abscisic acid action. - J. exp. Bot. 60: 1439-1463, 2009.

Spurr, A.R.: A low viscosity epoxy embedding medium for electron microscopy. - J. Ultrast. Res. 26: 31-43, 1969.

Taketa, S., Amano, S., Tsujino, Y., Sato, T., Saisho, D., Kakeda, K., Nomura, M., Suzuki, T., Matsumoto, T., Sato, K., Kanamori, H., Kawasaki, S., Takeda, K.: Barley grain with adhering hulls is controlled by an ERF family transcription factor gene regulating a lipid biosynthesis pathway. - Proc. nat. Acad. Sci. USA 105: 4062-7, 2008.

Tanaka, T., Tanaka, H., Machida, C., Watanabe, M., Machida, Y.: A new method for rapid visualization of defects in leaf cuticle reveals five intrinsic patterns of surface defects in Arabidopsis. - Plant J. 37: 139-146, 2004.

Tischner, T., Köszegi, B., Veisz, O.: Climatic programmes used in the Martonvásár phytotron most frequently in recent years. - Acta agron. hung. 45: 85-104, 1997.

Wang, Y., Wan, L., Zhang, L., Zhang, Z., Zhang, H., Quan, R., Zhou, S., Huang, R.: An ethylene response factor OsWR1 responsive to drought stress transcriptionally activates wax synthesis related genes and increases wax production in rice. - Plant mol. Biol. 78: 275-288, 2012.

Weng H., Molina I., Shockey J., Browse J. Organ fusion and defective cuticle function in a lacs1 lacs2 double mutant of Arabidopsis. - Planta 231:1089-1100, 2010.

Wu, R., Li, S., He, S., Wassmann, F., Yu, C., Qin, G., Schreiber, L., Qu, L.J., Gu, H.: CFL1, a WW domain protein, regulates cuticle development by modulating the function of HDG1, a class IV homeodomain transcription factor, in rice and Arabidopsis. - Plant Cell 23: 3392-3411, 2011.

Yang, J., Ordiz, M., Jaworski, J.G., Beachy, R.N.: Induced accumulation of cuticular waxes enhances drought tolerance in Arabidopsis by changes in development of stomata. Plant Physiol. Biochem. 49: 1448-1455, 2011.

Yu, D., Ranathunge, K., Huang, H., Pei, Z., Franke, R., Schreiber, L., He, C.: Wax Crystal-Sparse Leafl encodes a beta-ketoacyl CoA synthase involved in biosynthesis of 


\section{K. JÄGER et al.}

cuticular waxes on rice leaf. - Planta 228: 675-685, 2008.

Zhang J.Y., Broeckling C.D., Blancaflor E.B., Sledge M.K., Sumner L.W., Wang Z.Y.: Overexpression of WXP1, a putative Medicago truncatula AP2 domain-containing transcription factor gene, increases cuticular wax accumulation and enhances drought tolerance in transgenic alfalfa (Medicago sativa). - Plant J. 42: 689-707, 2005.

Zhang X., Henriques R., Lin S.S., Niu Q.W., Chua N.H.: Agrobacterium-mediated transformation of Arabidopsis thaliana using the floral dip method. - Nat. Protocols 1:641646, 2006. 